\title{
Distributed Auto-configuration of Neighboring Cell Graphs in Radio Access Networks
}

\author{
Javier Baliosian and Rolf Stadler
}

\begin{abstract}
In order to execute a handover processes in a GSM or UMTS Radio Access Network, each cell has a list of neighbors to which such handovers may be made. Today, these lists are statically configured during network planning, which does not allow for dynamic adaptation of the network to changes and unexpected events such as a cell failure. This paper advocates an autonomic, decentralized approach to dynamically configure neighboring cell lists. The main contribution of this work is a novel protocol, called DOC, which detects and continuously tracks the coverage overlaps among cells. The protocol executes on a spanning tree where the nodes are radio base stations and the links represent communication channels. Over this tree, nodes periodically exchange information about terminals that are in their respective coverage area. Bloom filters are used for efficient representations of terminal sets and efficient set operations. The protocol aggregates Bloom filters to reduce the communication overhead and also for routing messages along the tree. Using simulation, we study the system in steady state, when a base station is added or a base station fails, and also during the initialization phase where the system self-configures.
\end{abstract}

Index Terms-Self-management, distributed management, auto-configuration, radio access networks, UMTS.

\section{INTRODUCTION}

$\mathbf{T}$ HE demand for Internet access over public cellular networks has led to the creation of the Universal Mobile Telecommunication System (UMTS) [1], a third generation (3G) mobile networks technology, which integrates features of both IP and traditional telecommunications networks. A critical and complex issue with UMTS networks (or radio access networks in general) is their configuration, which is the focus of this work. Today, the configuration of these networks is a labor-intensive task. It requires special expertise, and it is carried out as part of the network planning process.

As reducing operational costs is a primary objective for operators, it is important to achieve a high degree of automation when configuring a network. In addition to reducing the costs of the planning process, there are further reasons for enabling auto-configuration in UMTS networks. They relate to the need of the system to dynamically adapt to changes in its environment, in order to maintain high levels of availability and efficiency. For example, with the proliferation of ever smaller cells, inside large buildings or even in homes,

Manuscript received August 3, 2009; revised January 22, 2010. The associate editor coordinating the review of this paper and approving it for publication was Nikolaos Anerousis.

J. Baliosian was with the Ericsson Ireland Research Centre, Athlone, Ireland during most of this work. Currently, he is with the School of Engineering, University of the Republic, Montevideo, Uruguay. (e-mail: javierba@ fing.edu.uy).

R. Stadler is with the ACCESS Linnaeus Center, KTH Royal Institute of Technology, Stockholm, Sweden. (e-mail: stadler@ee.kth.se).

Digital Object Identifier 10.1109/TNSM.2010.I9P0330. statically configuring these networks will become unfeasible and, therefore, automation must be (gradually) introduced to support re-configuration. Second, the system must be able to dynamically adapt its configuration, for instance, to a changing load pattern. Specifically, components may switch to standby mode and save energy when the current load can be handled by a subset of the available cells. Third, the system must be able to rapidly and automatically adapt to and recover from failures. This is important as temporary failures due to unreliable power supply are quite common in certain parts of the world.

This paper focuses on configuring and adapting a crucial data structure for call management, namely, the neighboring cell list (NCL), which controls the handover process of terminals between cells in a radio access network. Today, NCLs are generated as part of network planning and are configured statically in the Radio Network Controllers (RNCs). RNCs are entities that control large sets of Radio Base Stations (RBSs), often several hundreds. An RBS serves as a wireless access point to the network for terminals and includes several cells. RNCs and RBSs are entities of the Universal Terrestrial Radio Access Network (UTRAN), an overview of which can be found in [2].

In this paper, we introduce an autonomous method to configure and dynamically adapt the NCL for each cell in the network. The core of this method is a protocol that discovers, for every cell in the network, the set of cells with whom a given cell overlaps in radio coverage. We call this protocol DOC, for Discovering Overlapping Cells.

There are several approaches to discovering neighboring cells. Our protocol, DOC, assumes that each base station maintains a list of the terminals currently inside its coverage area. An advantage of making this assumption over other approaches (that rely on different assumptions) is that it facilitates the configuration of NCLs with cells of different providers and different technologies, but it comes at the expense of each RBS decoding the signals from all terminals in its coverage area. (See the related work section for details.)

Configuration of neighboring cell lists is performed in a centralized way in current UTRAN management systems. The framework and protocol we introduce in this paper, however, perform configuration in a decentralized way. The main reason for this choice is to enable rapid re-configuration in a large network.

The DOC protocol executes on an overlay with the topology of a spanning tree where the nodes are RBSs and the links represent communication channels. Over this tree, nodes periodically exchange the sets of terminals that are currently in their respective coverage area. We use Bloom filters to 
achieve efficient representations of terminal sets and efficient set operations. Bloom filters are aggregated to reduce the communication overhead and also for routing messages along the tree.

This paper contains a significant extension of an earlier work by us [3]. Compared to what is presented in [3], the DOC protocol has been improved, its evaluation is much more thorough, a baseline protocol has been introduced for the sake of comparison, and a more realistic simulation model has been adopted, taking into account terminal mobility and radio aspects. In addition, the related work and the discussion sections have both been expanded.

The paper is organized as follows. The next section outlines our framework for policy-based, autonomic configuration of neighboring cell lists. The framework includes three layers of functionality on which different (distributed) graphs are maintained. Section III presents DOC, the protocol that creates and maintains the graph of overlapping cells. Section IV evaluates the proposed protocol through simulations, and Section V reviews the related work. Finally, Section VI states the contribution of the paper and discusses future work.

\section{Autonomic CONFiguration OF NeIGHBoring CELLS}

The aim of this work is to engineer a system that automatically configures and maintains the neighboring cell list of each cell in a distributed and scalable manner. The framework depicted in Figure 1 shows our approach. It includes three planes, each of which contains functionality that maintains a graph associated with the plane. The lowest plane maintains the management overlay that provides transport services. This overlay has the topology of a spanning tree. There are many protocols available for building such trees on top of the existing IP-based management network that interconnects all base stations, for example [4].

For two cells to be configured as neighbors, they must fulfill a set of requirements, the most basic of which is that their respective coverage areas must overlap. For this reason, the second layer in our framework creates and maintains the graph of overlapping cells. Each node in this graph represents a cell, and each edge between two nodes means that the two cells overlap. Since the base stations corresponding to two overlapping cells may not be inside the radio-coverage area of each other (as seen in Figure 2), creating this graph of overlapping cells is not a straight-forward task. The protocol that implements this task is the focus of this paper, and is described in Section III.

The third layer in our framework builds the actual neighboring cells graph, which is a sub-graph of the overlapping cells graph on the second layer. The process of building this graph is policy-based. The detailed approach for this procedure is given in [5] and its implementation is planed for future work. Below are some sample policies for building the graph.

- A cell can have up to 32 intra-frequency neighbors.

- A cell can have up to 32 inter-frequency neighbors.

- A cell can have up to 32 inter-system (GSM) neighbors.

- Neighbor cells must have a high frequency of successful handovers.

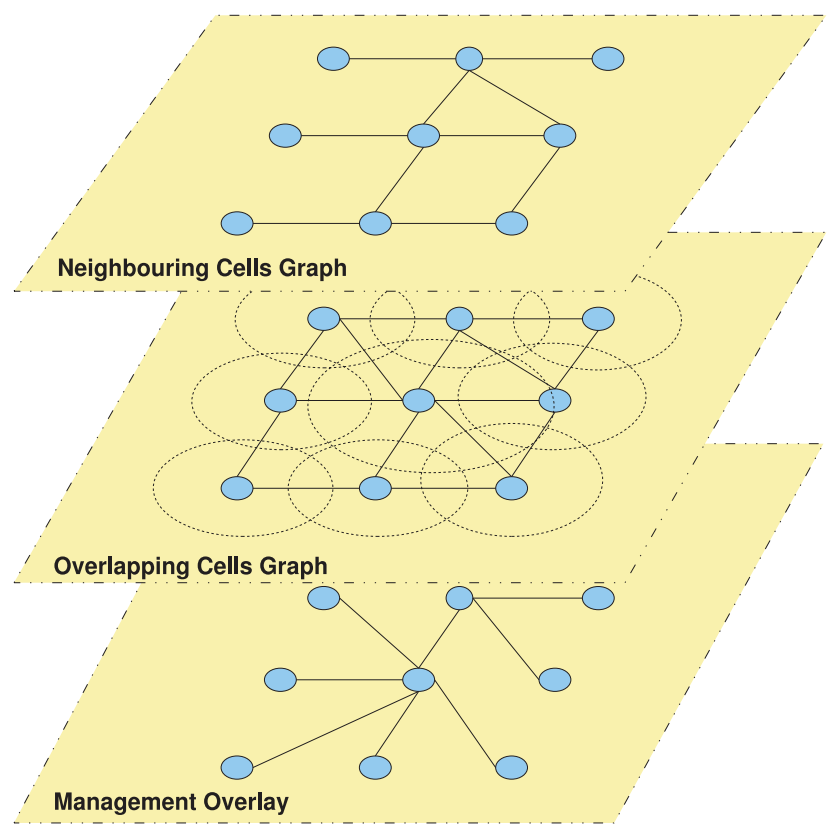

Fig. 1. Our Framework for Configuring the Neighboring Cell Lists. Three graphs must be maintained.

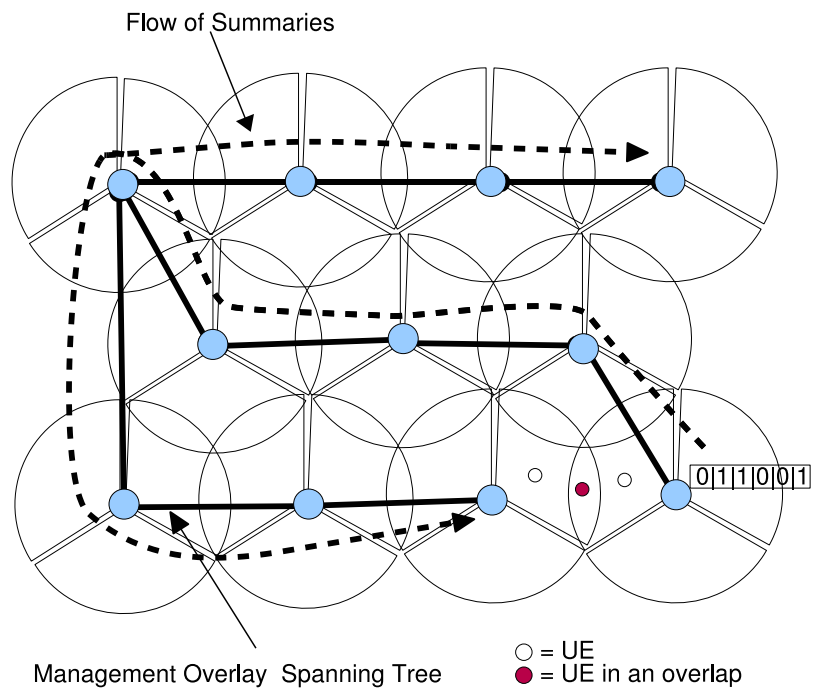

Fig. 2. An Example of Flooding Summaries on the Management Overlay.

\section{Discovering Overlapping Cells}

The basic idea behind our protocol for discovering overlapping cells (DOC) is simple: each base station in the network knows the terminals that are currently in range of its associated cells. (Terminals are also called User Equipments (UEs) in the UMTS context.) The base station ${ }^{1}$ floods the set of terminals in its range on a management overlay to all other base stations and then computes the intersection of its own terminal set with those received from other stations. Whenever the base station finds a sufficiently large intersection of its set of terminals with that of another station (as seen in Figure 2), it concludes that there is a coverage overlap between the two corresponding cells. Below, we introduce a protocol that implements this approach in a straightforward manner. It serves as a baseline

\footnotetext{
${ }^{1}$ Later on, we also use the term node for base station.
} 
protocol against which DOC will be evaluated in Section IV. Further, DOC can be understood as a refinement of this naive protocol.

\section{A. A Naive Protocol}

The design principle behind the naive protocol is simplicity. Its functional objective is to build and maintain, in a distributed fashion, the graph represented by the connectivity matrix

$$
\operatorname{Overlap}_{i j}(t)= \begin{cases}1, & \text { if }\left|U_{i}(t) \cap U_{j}(t)\right|>H, H \in \mathbb{N} \\ 0, & \text { else }\end{cases}
$$

where $U_{i}(t)$ is the set of UEs inside the cell $i$ at time $t$. We call two cells $i$ and $j$ overlapping, if the number of terminals in the intersection $U_{i}(t) \cap U_{j}(t)$ is larger than $H$. This threshold $H$ serves on the one hand as an hysteresis parameter to avoid oscillations, and on the other hand influences the degree of connectivity of $\operatorname{Overlap}_{i j}(t)$. Its optimal value must be determined experimentally.

We assume that the protocol executes on a management overlay that is a spanning tree (see Figure 1), and that a function is available on each node $j$ which provides the list of UEs in range $U_{j}(t)=\left\{u e_{j_{0}}, \ldots, u e_{j_{n}}\right\}$ when invoked at time $t$. Additionally, we assume an asynchronous reliable message service between nodes.

1) Node State: Here, we discuss the local state maintained by each node and its representation in the pseudocode in Figure 3.

Table of Overlapping Cells: this is the set of cells which a node believes that the local cell is currently overlapping with. Each element in the set is time-stamped when inserted. This set at node $j$ is represented as $\boldsymbol{O}_{\boldsymbol{j}}$ in the pseudocode.

Set of User Equipments in range: this is the set of the UEs (i.e., terminals) inside a given cell at a given time. In the pseudocode it is represented as $\boldsymbol{U}_{\boldsymbol{j}}(\boldsymbol{t})$ for the cell $j$ and time $t$.

Set of Management-Overlay Neighbors: this is the set of neighbors in the management overlay. In the pseudocode it is represented as $\boldsymbol{K}_{\boldsymbol{j}}$ for the node $j$. (Note that the concept of neighbor in the management overlay used here is different from the concept of neighbor in UMTS terminology.)

2) Types of Messages:

UE-list Message: this message is a tuple ue(forwarding_node, source, set_of_UE_ids) used to communicate an explicit set of UEs, where forwarding_node is the overlay neighbor that sent the message and source is the node that created set_of_UE_ids. This message has a variable length depending on the size of set_of_UE_ids. An UE-id has 16 bits, therefore an UE-list message has a minimum of 16 bits for one UE-id, plus 32 bits for the forwarding_node address, 32 more for the source address and 8 bits for the header, making a message with a minimum size of 88 bits.

Overlapping Message: this is a message overlap(node_id) that communicates a confirmed overlapping relationship. This message has a fixed size of 40 bits.

3) Pseudocode: Figure 3 shows the pseudocode of the naive protocol. It is structured as two local processes that run asynchronously and independently on each node. Naive
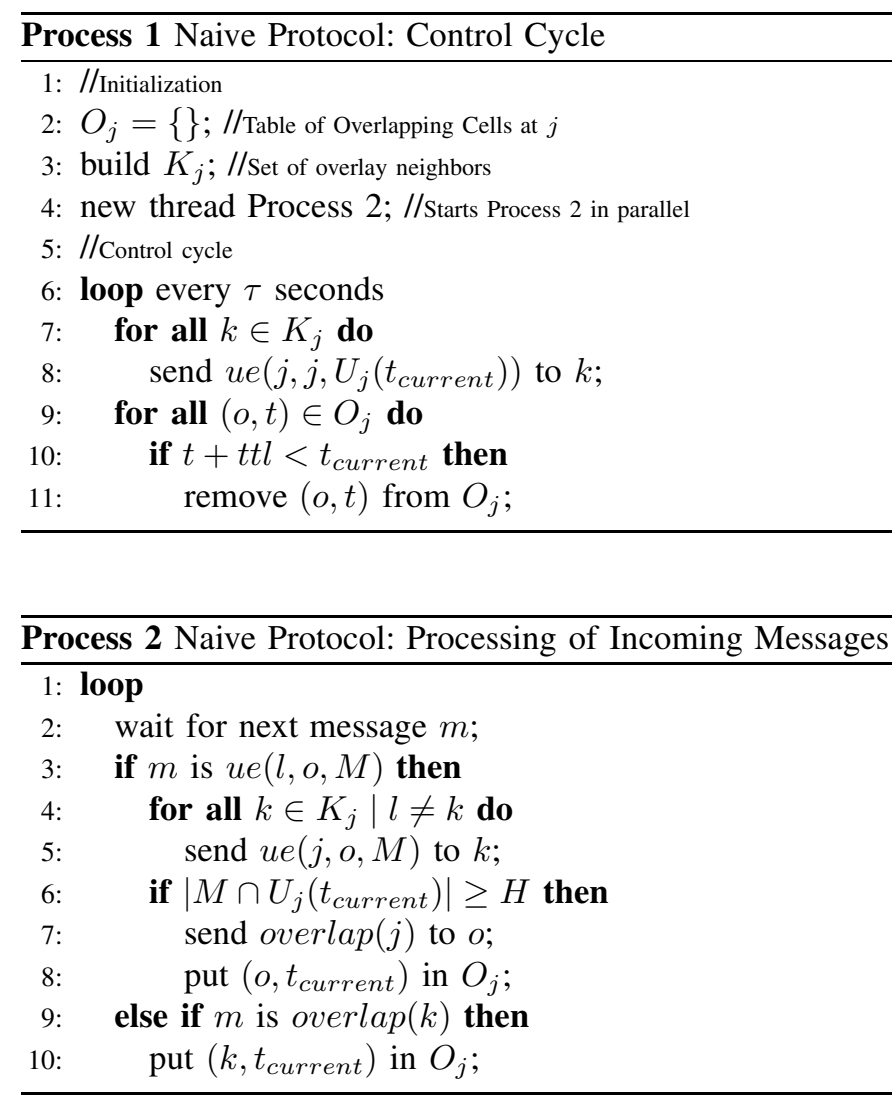

Fig. 3. Pseudocode for Naive Protocol on Node j

Protocol Process 1 describes the local control cycle of the protocol, which is periodically executed on each node.

The loop in Naive Protocol Process 1 is executed every $\tau$ seconds. First, the set of terminals in range is determined and sent to each neighbor $k$ on the spanning tree (line 8). Second, the node removes those entries from the set of overlapping cells that have not recently been refreshed (lines 9-11).

Naive Protocol Process 2 describes how a node processes messages that it receives from its FIFO input queue.

Upon receiving a ue-list message, the node computes the intersection between its local terminal set and the received terminal set. If this intersection is sufficiently large, an overlapping message is sent back to the originator of the received ue-list message. In addition, the node adds the originator of the ue-list message to its set of overlapping cells (lines 6-8). Upon receiving an overlapping message, the node adds the originator of the message to its set of overlapping cells (line 10).

The control parameters of this algorithm are the control period $\tau$, the intersection threshold $H$ for considering two cells to be overlapping and, the time to live (ttl) after which a reference to an overlapping cell expires.

Note that this naive protocol can be further simplified. For example, overlapping messages are not strictly necessary, since we can argue that all cells can determine whether they overlap with other cells, by just comparing ue-sets. However, since UEs are mobile, this may lead to non-symmetric 


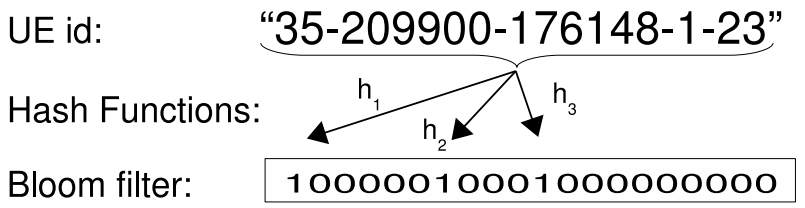

Fig. 4. Constructing a Bloom Filter.

overlapping relationships between cells, something we want to avoid in the design of the DOC protocol (Section III-C). Therefore, we introduced the overlapping messages in the naive protocol, to make it produce, at any time, the same graph of overlapping cells as the DOC protocol. A similar argument applies to the $t t l$ parameter.

\section{B. Controlling Overhead}

Summarizing Sets: Although the graph of overlapping cells produced by the naive protocol, presented above, has the desired characteristics, this protocol leads to two problems: first, the flooding of the entire set of terminals that each cell has in range causes a large communication overhead. Second, the complexity of computing the intersection of two sets is of the order $O(n \times \log (n)+m \times \log (m))$, where $n$ and $m$ are the number of elements in each set. This may induce a prohibitive computational overhead on each node when the number of terminals in the network is very large.

We address these problems using Bloom filters, an efficient, probabilistic technique for representing and manipulating sets. We introduce Bloom filters below, and then we describe the proposed protocol in detail.

A Bloom filter [6] is a memory-efficient way of representing a set in an array of bits with the purpose of testing whether or not an element is member of the set. More precisely, a Bloom filter is a bit array of size $l$ and includes $f$ different hash functions, each of which maps a key value to one of the $l$ array positions. The filter is initialized with all bits set to 0 . To add an element to a filter, the element's key is hashed with each of the $f$ hash functions, each hash function produces an array position that is set to 1 (see Figure 4).

To test whether an element is a member of the set represented by a Bloom filter, its key is hashed with each of the $f$ hash functions to obtain $f$ array positions. If any of these positions has bit value 0 , the element is not in the set. If all have value 1 , the element is in the set with a certain probability. The reason why the element might not be in the set -which is called a false positive- is because the same bits might have been set to 1 during the insertion of other elements.

A discussion of the relationship of the probability between false-positives on one hand and the filter size and the number of hash functions on the other hand can be found in [7]. In essence, the higher the number of elements that are summarized in a given bit array, the higher the probability of a false positive. Further, for a given number of elements, one can determine a pair of array size and number of hash functions, such that the resulting Bloom filter exhibits a "reasonable" low probability for false positives.

An interesting property of Bloom filters is that the Bloom filter of the union of two sets can be computed as the Boolean
OR between the Bloom filters of each set. Similarly, the Bloom filter of the intersection of two sets is the Boolean AND between the Bloom filters of each set [6].

Additionally, it is possible to define the concept of distance between Bloom filters. The distance $|a-b|$ between two Bloom filters $a$ and $b$ of the same size, is defined as the number of bits that $a$ has different to $b$ normalized to the size of the Bloom filters.

Reducing Computational Overhead: As explained above, computing a (deterministic) intersection between two sets takes $O(n \times \log (n)+m \times \log (m))$, operations where $m$ and $n$ are the sizes of both sets. However, if the second of those sets is represented as a Bloom filter, computing a probabilistic intersection between these two sets takes $O(k \times m)$ operations because to perform this intersection we test for membership each of the $m$ elements in the first set against the Bloom filter.

Reducing Traffic Overhead: In our approach, the summarized sets of terminals are flooded on the network using a spanning tree on the management overlay.

By aggregating messages from different nodes, we reduce the number of messages, and performing this aggregation using Bloom filters union we keep the size of those messages constant. Both strategies together reduce the traffic overhead compared to a scheme in which each node broadcasts its set of terminals independently.

\section{The DOC Protocol}

In this subsection, we describe the design goals of the protocol, the design principles that are behind the realization of the protocol, and we give a description of the protocol, which includes assumptions, the node state, the message types, and the pseudocode.

1) Design Goals: The DOC protocol has the same functional goal as the naive protocol presented in III-A.

In terms of performance, the primary goal of the DOC protocol is a short reaction time to external events, such as node failures and recovery, while keeping a low overhead. The second goal is that the traffic and computational overhead of the protocol must scale with both the network size and the number of terminals in the network.

2) Design Principles: the principles driving the DOC protocol design were (i) decentralization to achieve fast update of the overlapping cells graph, and (ii) probabilistic representation and aggregation of terminal sets to reduce overhead.

3) Protocol Overview: Every node disseminates information along the spanning tree in two ways. First, a node, periodically and if significant change has happened, sends a summary of its terminal set to its neighbors, which, in turn, aggregate this information with summaries of their neighbors and disseminate the resulting summary in the same way (see Figure 5). Bloom filters are used to produce the summaries and for the aggregation process.

Second, in response to a summary that a node A has received from a neighbor, it returns the probabilisticallycomputed intersection of its own terminal set with that of the summary. The neighbor then uses its summary table to route that information towards other nodes that may be overlapping with node A. The probabilistically-computed intersections are 


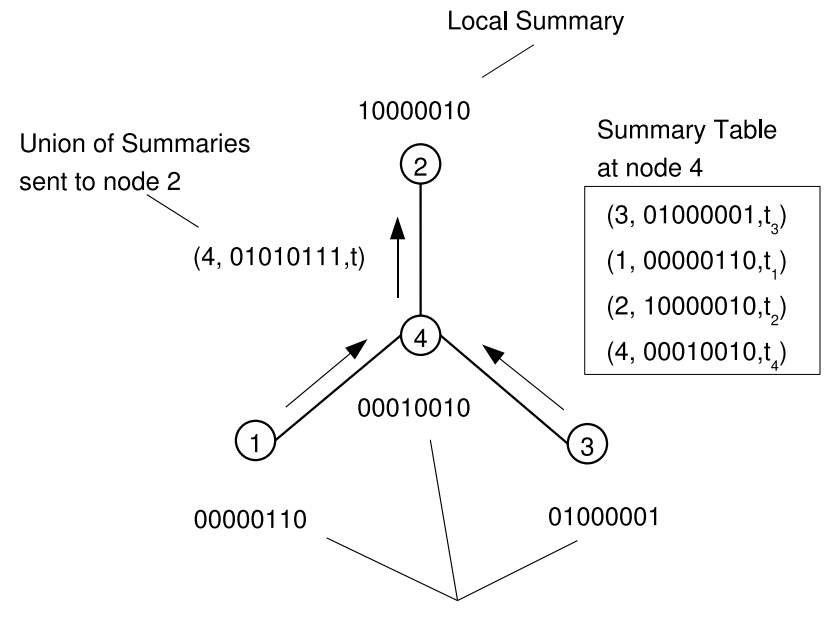

Local Summaries

Fig. 5. Aggregating Summaries on a Node.

validated by using deterministic intersection of terminal sets at the nodes that originated the summaries. These two ways of exchanging information are designed for a node to detect other nodes with jointly overlapping cells. All node state expires using a soft state concept, which allows the protocol to deal with cases where overlaps disappear.

The protocol uses Bloom filters to obtain an efficient probabilistic guess about which cells may overlap. Then, it confirms or refutes a positive guess by computing the intersection of candidate sets in a deterministic manner. Note that, due to using Bloom filters, the probabilistic part of the protocol does not produce false negatives. Therefore, the protocol computes accurately the set of overlapping cells.

4) Protocol Assumptions: The assumptions are the same as those made for the naive protocol (Section III-A), this is: the protocol operates on a management overlay that is a spanning tree (see Figure 1), and there is a function available on each node $j$ that provides the list of UEs in range $U_{j}(t)=\left\{u e_{j_{0}}, \ldots, u e_{j_{n}}\right\}$ when invoked at time $t$. Finally, we assume an asynchronous reliable message service between nodes.

5) Node State: In this subsection, we discuss the local state maintained by each node and its representation in the pseudocode in Figure 6. The DOC protocol shares with the naive protocol the Table of Overlapping Cells, the Set of User Equipments in range, and the Set of Management-Overlay Neighbors. In order to use Bloom filters for reducing the protocol overhead, we introduce two new tables.

Summary Table: this is the table of the received Bloom filters. Each entry has the format $(k, b, t)$ where $k$ is the neighbor that sent the Bloom filter $b$ and $t$ is the local timestamp when the entry was added. For each neighbor there is at most one entry in the summary table.

The summary table at node $j$ is represented as $\boldsymbol{S}_{\boldsymbol{j}}$ in the pseudocode.

Summary Table from the previous control cycle: this table is a copy of $\boldsymbol{S}_{\boldsymbol{j}}$ from the last control cycle. Its purpose is to reduce the protocol overhead by avoiding aggregating summaries when nothing has changed on them since the last cycle. This table at node $j$ is represented as $\boldsymbol{S}_{j}^{\prime}$ in the pseudocode.

6) Types of Messages: The DOC protocol shares with the naive protocol the ue-list Message and the Overlapping Message. In addition, we create the following type of message.

Summary Message: this message is a tuple sum(node_id, bloom_filter, time_stamp) used for communicating summaries of UE sets, where node_id is the sender of the message and time_stamp is the message creation time at the sending node. This message has a configurable fixed size. Its size is determined mainly by the size of the bloom_filter, therefore, a Summary message with a Bloom filter of 30,000 bits has a size of around 3.7KB.

\section{Pseudocode}

Figure 6 shows the pseudocode of the DOC protocol. It is structured in two processes which run asynchronously and independently on each node. DOC Process 1 describes the local control cycle of the protocol which is periodically executed on each node.

DOC Process 1 is a loop that is executed every $\tau$ seconds. First, the set of terminals in range is determined, encoded in a Bloom filter and inserted in the Summary Table (lines 11-14).

The local Bloom filter at the Summary Table is overwritten with a new one if the distance between the new one and the old one is more than $\mathrm{D}$, or if the old one is older that $t t l$ seconds (lines 13 and 14). Lines 15 to 21 describe the aggregation and forwarding process. For each neighbor $k$ on the spanning tree, the node aggregates those entries in the summary table that have changed since the previous control cycle and sends the aggregate to $k$ if changes have occurred. Next, the state of the summary table is saved (line 22). Finally, the node removes those entries from the set of overlapping cells that have not recently been refreshed (lines 23-25).

DOC Process 2 describes how a node processes messages that it receives from its FIFO input queue. Upon receiving a summary message, the node computes the (probabilistic) intersection between its terminal set and the received summary. If the intersection is sufficiently large, then the intersection set is returned to the neighbor who sent the summary (lines 311). Upon receiving a message with an intersection set (ue-list message), the node computes the (probabilistic) intersection between the received intersection set and each of the summaries in its summary table. For each case, if the resulting intersection set is sufficiently large, then the node sends this intersection set to the corresponding neighbor (lines 1320). Also, the node computes the (deterministic) intersection between the received intersection set and its terminal set. If this intersection is sufficiently large, an overlapping message is sent back to the originator of the received ue-list message. In addition the node adds the originator of the ue-list message to its set of overlapping cells (lines 21-23). Upon receiving an overlapping message, the node adds the originator of the message to its set of overlapping cells (line 25).

The control parameters of this algorithm are the control period $\tau$, the Bloom filter parameters, the distance between Bloom filters $D$, the intersection threshold $H$ for considering two cells to be overlapping and, the time to live (ttl) after which the node state variables expire. 

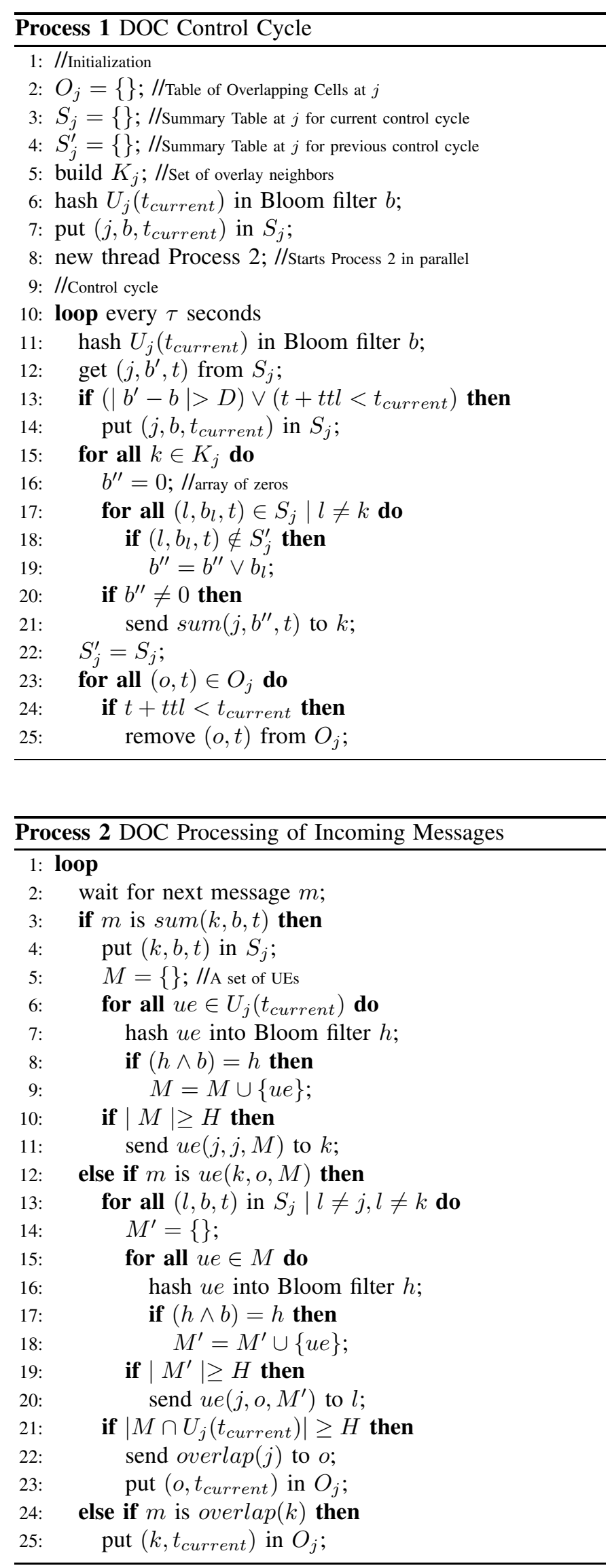

Fig. 6. Pseudocode for DOC Protocol on Node $\mathrm{j}$

\section{ExPERIMENTAL EVALUation}

The experimental evaluation in this section focuses on the scalability properties of the DOC protocol, the dimensioning of the protocol parameters for given network scenarios, and the behavior of the protocol during the initialization phase, as well as under system changes or failures.

In order to obtain a realistic evaluation, we choose three separated simulation packages, which, in combination and through interaction, create an environment that takes into account the dynamic aspects of radio coverage, terminal mobility and the execution of our protocol.

First, for simulating DOC and the required network entities, we use JavaSimulation [8], a discrete event systems simulation package. Second, the radio aspects of the access network and the terminals are simulated using NSWP [9], a tool that is programmed in Matlab. The simulated network is an UTRAN and the propagation model used is the Okumura-Hata with all radio parameters set to NSWP defaults. The interface between JavaSimulation and NSWP is written using JMatLink [10]. Third, the mobility of the terminals is simulated using BonnMotion [11], a library that supports a range of mobility patterns that have been studied in the recent literature.

In our simulations, Bloom filters are computed using the hash functions RS, JS, PJW, ELF, BKDR, SDBM, DJB, DEK and AP. They are described and available for download at [12].

\section{A. Evaluation Setup}

1) Network: The setup common to all the experiments is a network of RBSs, distributed as in Figure 2, where each RBS has three $90^{\circ}$ antennas that are set up at a $120^{\circ}$ angle from each other. As common in current deployments, each RBS controls three cells. In our simulations we run each RBS as a node and use a straight-forward extension of the DOC protocol described in Section III to take into account that a node has three cells. We report on experiments with networks of different sizes and different terminal densities (i.e., the number of terminals per cell).

2) Mobility Model: We assume an urban environment and use the Manhattan mobility model [13] to capture the movements of the terminals. Each terminal moves along a mesh of parallel, perpendicular streets placed every $150 \mathrm{~m}$. For all the experiments, the velocity of a terminal varies randomly with a mean of $8 \mathrm{~m} / \mathrm{s}$ and a standard deviation of $0.2 \mathrm{~m} / \mathrm{s}$. At each intersection, a terminal decides whether to turn or keep the direction with a probability of 0.5 .

3) Simulation Parameters: Each simulation run lasts $400 \mathrm{~s}$ simulation time. The system starts with empty summary tables $S_{j}$ and tables of overlapping cells $O_{j}$ and discovers the overlapping cells graph. For the experiments in steady state measurements are taken between 300s and 400s simulation time.

The DOC control parameters are set as follows: the protocol cycle $\tau$ is $5 \mathrm{~s}$ and the time to live is $30 \mathrm{~s}$. The Bloom filter parameters are set to an array size of 30,000 (bits) and 2 hash functions, except for the study of dimensioning the Bloom filter. The threshold $H$ is set to 10 (terminals) and the distance between summaries $D$ is set to 0.002 , except for the study of dimensioning of these parameters. 


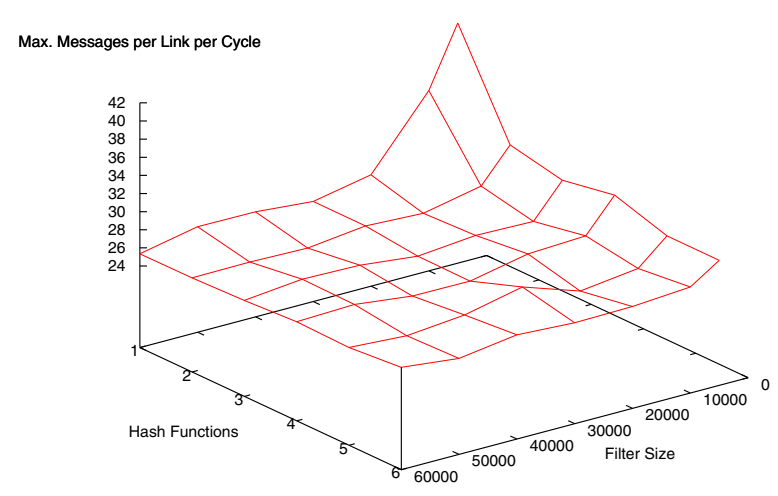

Fig. 7. Traffic Overhead vs. Bloom Filter Parameters (filter size, number of hash functions).

4) Management Overlay: The management overlay used for the simulation is a balanced binary tree. The communication delay for a message across a link is $4 \mathrm{~ms}$.

5) Evaluation Metrics: Depending on the particular experiment, we compute different metrics. We estimate the protocol overhead by measuring the maximum and the average number of messages per link per cycle (on the management overlay). As an indicator of the system dynamics, we measure the number of changes of the overlapping cells tables $O_{j}$ per node per cycle.

\section{B. Dimensioning of DOC Parameters}

The parameters for the Bloom filter, as well as $H$ and $D$ must be properly chosen to achieve a system that exhibits low protocol overhead, is stable in steady state and produces a graph of overlapping cells is close to reality.

1) Bloom Filter Dimensioning: First, we study how Bloom filter parameters impact the DOC traffic overhead. Specifically, we investigate how the combination of filter size and the number of hash functions influences the number of messages per link per cycle. The experiments are set up as described above. The network size is 57 cells with 100 terminals per cell, on a field of $12,000 \times 12,000 \mathrm{~m}$.

Figure 7 shows the simulation results. Each point on the graph represents an average over 20 runs of the same experiment. We observe that, as the size of the Bloom filter increases (which reduces the probability of false positives), the maximum number of messages per link decreases monotonically. Similarly, increasing the number of hash functions (which, in the range considered, also reduces the probability of false positives) decreases the maximum number of messages per link, although this relationship is less visible on the graph. Our conclusion is that, for this particular setup, a filter size between 10,000 and 30,000 and 2 to 4 hash functions define an operating region with a low overhead, while keeping the message size relatively small (between $1.2 \mathrm{~KB}$ and $3.7 \mathrm{~KB}$ ) and also keeping low the computational overhead of hashing terminal identifiers.

2) Dimensioning of Distance $(D)$ and Threshold $(H)$ : The purpose of this series of experiments is to study how the parameters distance $(D)$ and threshold $(H)$ impact the DOC

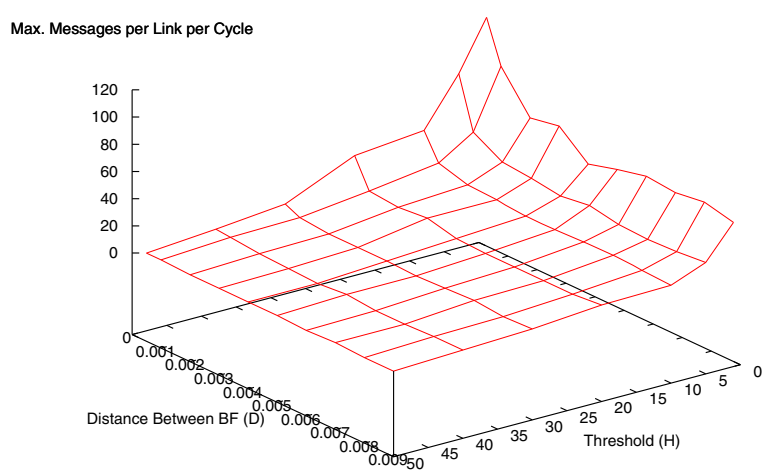

Fig. 8. Traffic Overhead vs. Distance Between Two Bloom Filters (D) and Threshold of the Size of the Intersection Between Terminal Sets $(\mathrm{H})$ (see Section III-D).

Changes in the Overlapping Cells Tables

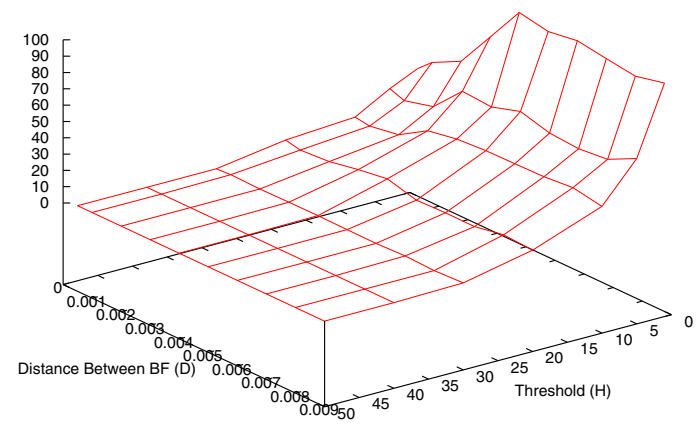

Fig. 9. Changes in the Tables of Overlapping Cells vs. Distance Between Two Bloom Filters (D) and Threshold of the Size of the Intersection Between Terminal Sets $(\mathrm{H})$ (see Section III-D).

traffic overhead and the stability of the graph of overlapping cells, for a system in steady state. Specifically, we investigate how the combination of $D$ and $H$ influences the number of messages per link per cycle and the total number of changes in the tables of overlapping cells.

The experiments are set up as described above. The network size is 57 cells with 100 terminals per cell, on a field of $12,000 \times 12,000 \mathrm{~m}$.

Figures 8 and 9 show the results. Each point on the graph represents an average over 20 runs of the same experiment. In Figure 8, we observe that, as the parameter $D$ increases (which reduces the update rate of local summaries), the maximum number of messages per link per cycle decreases monotonically. Similarly, increasing the threshold $H$ (which decreases the probability that two cells overlap), decreases the maximum number of messages per link. Figure 9 shows that the impact of parameter $H$ on the stability of the Table of Overlapping Cells (or state stability) is qualitatively the same as its impact on the overhead. However, the parameter $D$ influence the state stability in a different manner. Increasing $D$ up to a certain value results in an increasing number of changes. Beyond that value, the number of changes declines. We explain this 
behavior with the fact that the Tables of Overlapping Cells are soft state. As the update rate of local summaries decreases, there comes a point beyond which entries in the Table of Overlapping Cells expire. They are then removed from the table and later reinserted by the protocol, which causes the system to oscillate.

Our conclusion from these experiments is that, for this particular setup, a value $D$ of around 0.002 and a threshold $H$ of around 10 define an operating region with a low protocol overhead while keeping the number of changes very low.

\section{Scalability With Respect to the Network Size and Terminal Density}

In this subsection, we present the results of four sets of experiments designed to study the scalability properties of the DOC protocol and how those compare to the performance of the naive protocol presented in Section III-A, which we use as a baseline protocol. First, we study the relationship between the number of cells in the network and the overhead of the DOC protocol for a system in steady state. In this experiment, we simulate 100 terminals per cell and use the following settings: $D=0.002, H=10$; the Bloom-filter parameters are set to a filter size of 30,000 and 2 hash functions; the number of cells in the network varies from 18 to 243 . The remaining setup is described in Section IV-A.

Figure 10(a) shows the result of the simulations. Each point on the graphs represents an average over 10 runs of the same experiment. We make two observations. First, the maximum overhead (measured in messages per link per cycle) is significantly larger than the average overhead. We explain this difference by the tree topology of the overlay. A large number of ue-list messages is routed through the root, which causes the relatively high overhead on links close to the root. Possible solutions for better balancing the load will be discussed in Section VI.

Second, we notice the influence of the size of the Bloom filter on the protocol overhead. A Bloom filter of 60.000 bits produces a lower overhead than a Bloom filter size of 30.000 bits, as a larger filter size reduces the probability of false positives and, therefore, the number of UE-list messages. As expected from earlier experiments, the maximum overhead for a filter of 60,000 bits remains approximately constant within the ranges of system sizes investigated, while the overhead increases with a filter size of 30,000 bits. We conclude that for system sizes of up to 243 cells a filter size of 60,000 bits is sufficient.

With the second set of experiments presented in this subsection, we study the impact of the number of terminals in the network on the DOC protocol overhead for a system in steady state. We use the following settings: $D=0.002, H=10$; the Bloom-filter parameters are set to a filter size of 30,000 and 2 hash functions; the number of terminals per cell varies from 20 to 280 , and the number of cells is 57 . The remaining setup is described in Section IV-A.

Figure 11(a) shows the results. Each point on the graphs represents the outcome of one simulation run.

We observe that the protocol overhead increases linearly with the terminal density. We believe that the main contributor

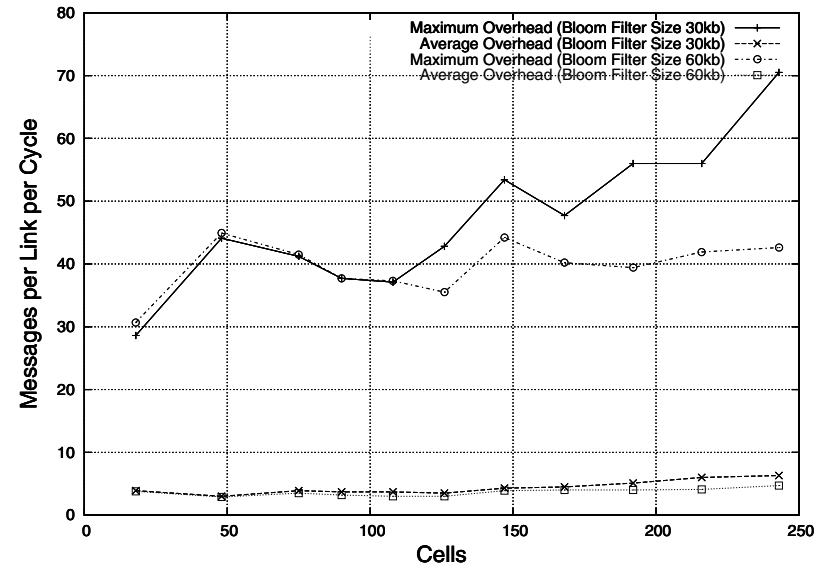

(a) DOC Overhead vs. System Size.

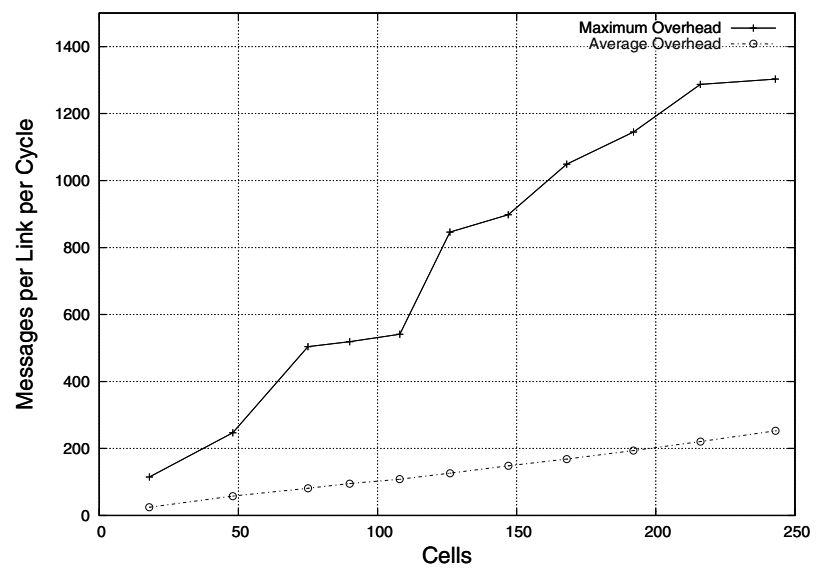

(b) Naive Protocol Overhead vs. System Size.

Fig. 10. Overhead Comparison between DOC and the Naive Protocol in Steady State. Density is 100 Terminals per Cell.

to this increase is the fact that the number of cells found to be overlapping increases with the terminal density, as a result of our definition of when two cells overlap.

Since the literature does not provide us with sufficient information that would allow us to evaluate DOC against alternative strategies (see Section V), we developed the baseline protocol presented in Section III-A to investigate the performance gain of DOC compared with a naive approach. The third and fourth sets of experiments, depicted in figures 10(b) and 11(b), are the result of this comparison.

Figure 10(b) shows the result of a set of experiments in which the naive protocol is run with the same simulation setup used for the experiments with DOC depicted in Figure 10(a). We observe that the naive protocol exhibits an overhead that is at least one order of magnitude larger than the DOC protocol (using well-dimensioned Bloom filters), and that the overhead increases linearly with the system size. Both of these observations are expected, since the naive protocol does not aggregate terminal sets when they are sent along the management overlay.

Figure 11(b) shows the result of a set of experiments in which the naive protocol is run with the same simulation setup used for the experiments with DOC depicted in Figure 11(a). As in the previous experiment, the overhead of the naive protocol is at least one of magnitude higher than the number 


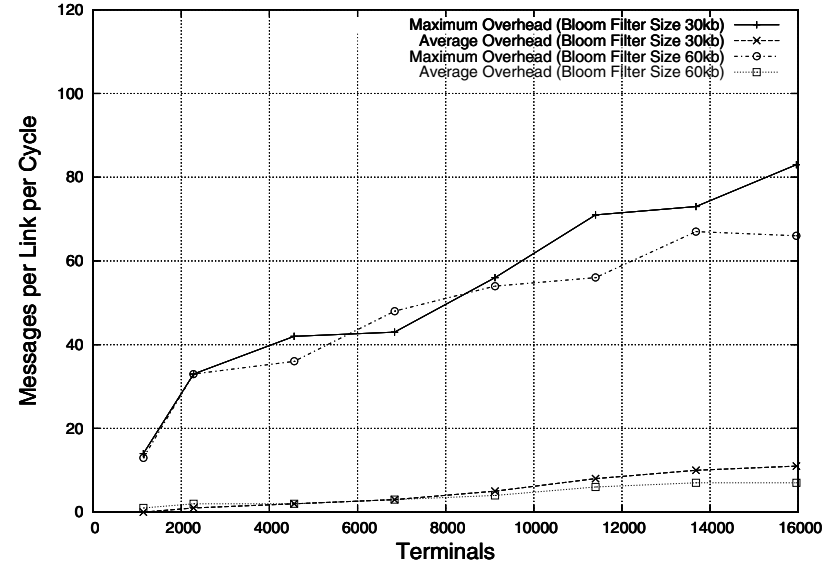

(a) DOC Overhead vs. Terminal Density.

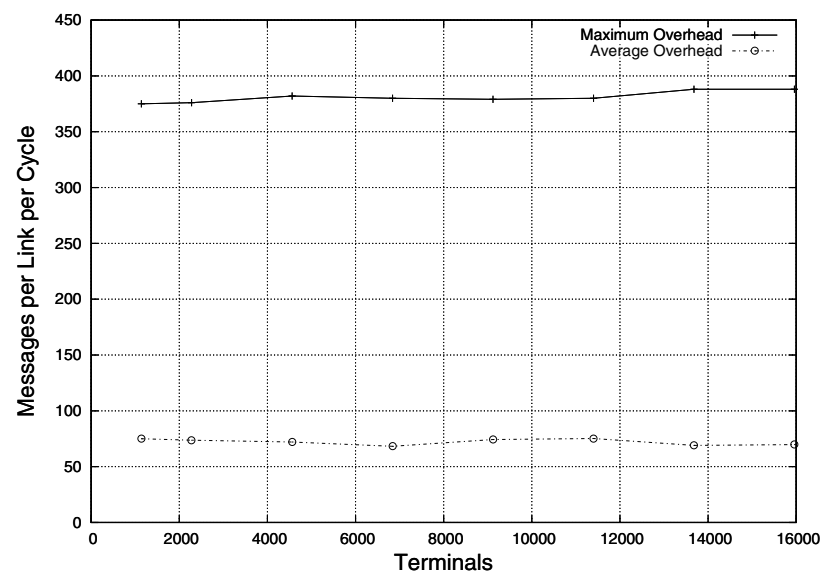

(b) Naive Protocol Overhead vs. Terminal Density.

Fig. 11. Overhead Comparison between DOC and the Naive Protocol in Steady State. System Size is 57 Cells.

of messages produced by the DOC protocol. As expected, the naive protocol overhead, in terms of number of messages, is independent of the terminal density, since the system size remains constant. However, while the size of the summary DOC messages is constant, the message size for the naive protocol increases linearly with the terminal density, a fact that is not captured in Figure 11(b).

We conclude that, compared with the baseline protocol, DOC has a significantly lower overhead.

\section{Self-configuration During Initialization Phase}

The purpose of this series of experiments is to study the system during its initialization phase. Specifically, we are interested in the time it takes for the system to reach a steady state, i.e., when the Table of Overlapping Cells has become stable. In this experiment, we simulated three different system sizes: 27, 75 and 192 cells, all with a density of 100 terminals per cell and we use the following settings: $D=0.002, H=10$; the Bloom-filter parameters are set to a filter size of 30,000 and 2 hash functions. The remaining set up is described in IV-A.

Figure 12 gives the average number of DOC messages per link over time. Each curve in this graph is the result of averaging over 10 runs of the same experiment. All three

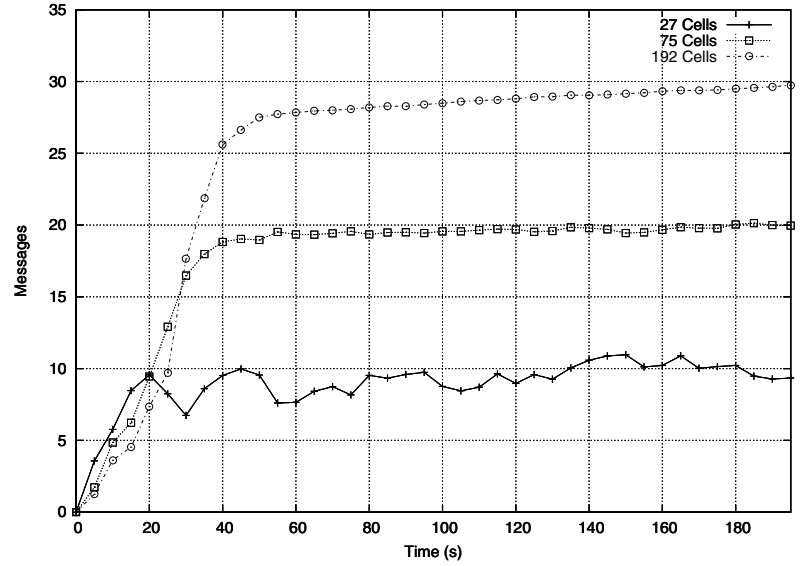

Fig. 12. DOC Overhead during System Initialization. Graphs show average message rate per link. The length of the protocol cycle is $5 \mathrm{sec}$.

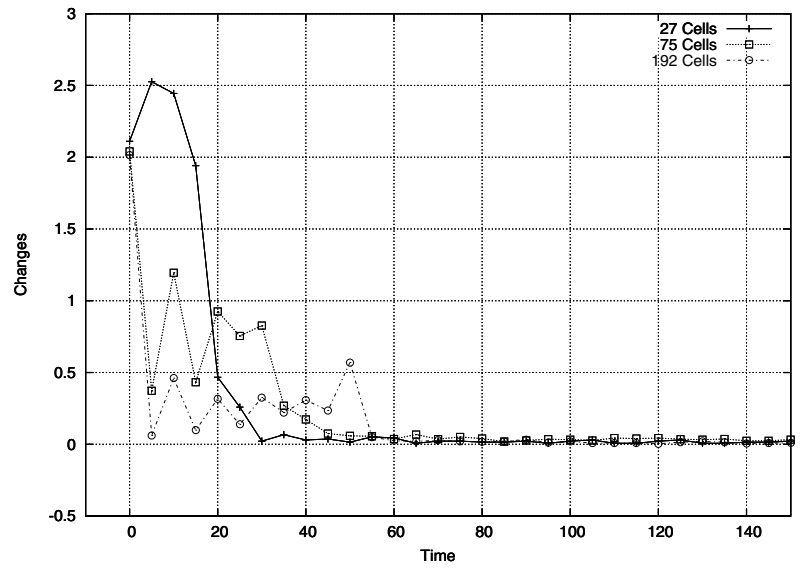

Fig. 13. Change Rate for Tables of Overlapping Cells during Initialization. The length of the protocol cycle is $5 \mathrm{sec}$.

curves converge towards the steady state with an overhead that is consistent with the measurements given in Figure 10(a). The graphs suggest that the smaller the system size, the shorter the convergence time.

Figure 13 shows the evolution of the change rate (i.e., number of the changes per node during each protocol cycle) over time for the Table of Overlapping Cells. As above, each curve in this graph is the result of averaging over 10 runs. As expected, the smallest system has the shortest convergence time, and the largest system has the longest. After 60s, which corresponds to 12 cycles, all the systems exhibit only occasional changes.

We conclude from these experiments that it is possible to initialize the protocol and reach steady state in very few cycles (roughly twice the height of the overlay tree), even for systems with some 200 cells.

\section{E. Adaptation to Node Addition and Node Failure}

We study the rate of change and time that DOC takes to re-configure the Tables of Overlapping Cells in response to events, such as the addition of an RBS (with three cells) or the failure of an RBS. In this experiment, we simulate 57 cells with 100 terminals per cell and we use the following settings: 


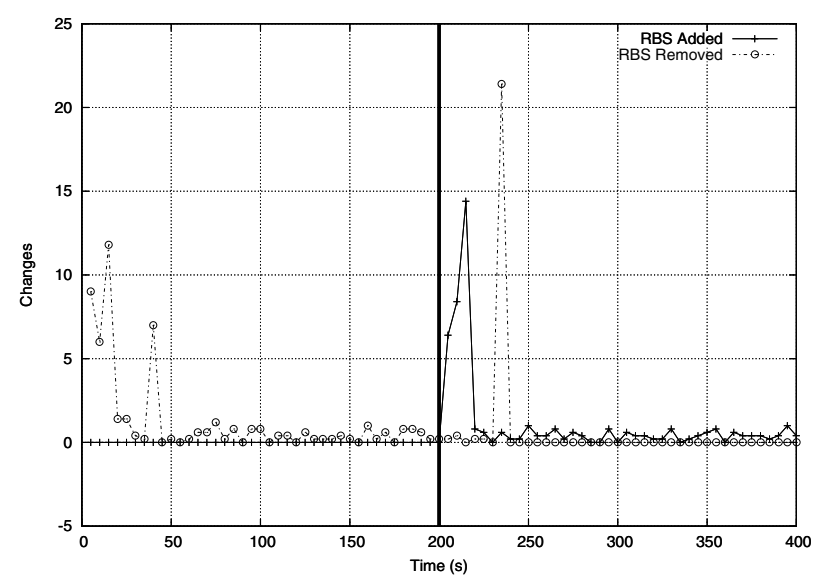

Fig. 14. Change Rate for Tables of Overlapping Cells during Node Addition and Node Failure. The addition or the failure occurs at 200s simulation time. Only those changes that relate to the affected node are considered.

$D=0.002, H=10$; the Bloom-filter parameters are set to a filter size of 30,000 and 2 hash functions. The remaining setup is described in Section IV.

Figure 14 shows the graphs of two simulation experiments. In the first experiment, an RBS (which includes three cells) is added at time 200s, while the system is in steady state. In the second experiment, an RBS with three cells fails at time 200s. Both curves in the figure are the result of averaging the measurements over five runs each.

We observe that the adaptation process is triggered immediately after adding an RBS, while the response to an RBS failure is delayed. This behavior is a direct consequence of the protocol design, where the added node immediately starts advertising its summary, while the failure of a node is detected only after its entry in the Tables of Overlapping Cells of other nodes expires after a $t t l$ period of 30 s.

The time needed for a cells to detect a new overlapping cell depends on the height of the overlay spanning tree and the control period $\tau$. In the worst case, and disregarding communication delays, if the height of the spanning tree is $g$, the summary of the set of terminals sent by a cell $A$ takes $2 \times g \times\left(\tau+\right.$ processing_time $\left.{ }_{1}\right)$ seconds to arrive and be processed by any other cell $B$. The UE-list message sent back by $B$ takes $2 \times g \times$ processing_time 2 seconds to arrive at $A$. Therefore, disregarding the time needed to send the overlap message directly from $A$ to $B$, the maximum time needed to detect a new overlapping cell is:

$2 \times g \times\left(\tau+\right.$ processing_time $e_{1}+$ processing_time $\left.e_{2}\right)$ seconds

Additionally, note that the protocol overhead for adding a node is small, and there is no overhead associated with a node failure.

\section{RELATED WORK}

There exists an extensive body work on topology discovery for ad-hoc networks, for example [14] and [15]. The methods described in this literature assume that two neighbors in the topology graph are base stations in range of each other. However, this is generally not the case for neighboring base stations of cellular networks. Therefore, these methods are not appropriate for the work in this paper.

Chapter 9 of [9] contains a proposal for automatic configuration of neighboring cells. The authors present a centralized, iterative method that uses handover statistics. If these statistics between two cells show a small drop-rate, then the cells are likely kept as neighbors. The proposal concentrates on building and maintaining the neighboring cells graph depicted in Figure 1 and takes the graph of overlapping cells, which our method aims to discover, as given. In this sense, the work in [9] is complementary to work presented in this paper.

Olofsson et al. made one of the first contributions to the automatic configuration of the list of neighbors of a cell in GSM networks [16], [17]. Their strategy is to add a random set of cells to the current neighbor list of a cell. This makes the terminals connected to a cell measure the signal from additional cells. The signal quality of the newly added cells and hand-over statistics are used then to choose a new list of neighbors for a given cell. Like the work described above, this work assumes the existence of an overlapping cells graph, and, therefore, is complementary to the research presented in this paper.

Parodi et al. [18] proposed a method for the definition of neighboring cell lists, in which the overlapping relationships are computed based on estimating the cell coverage areas. Two cells are considered neighbors if their coverage areas overlap. The coverage areas are estimated using antenna and wave propagation models. The effectiveness of this method highly depends on how accurately these models reproduce the propagation properties of a specific terrain. In our work, we measure the overlap between two cells through the existence of terminals in the joint coverage areas, and we do not estimate the overlap using propagation models, as the authors in [18] propose.

Soldani and Ore performed research on configuring neighboring cell lists for UMTS networks, whereby the terminals detect new cells in their vicinity and report them to the Radio Network Controller [19]. The detected cells are ranked using performance metrics, and the best cells are chosen as neighbors. The method proposed in [19] is specific to UMTS networks and is not suitable for earlier technologies including GSM. The approach in [19] discovers overlapping cells through using terminals as sensors, while in our work overlaps are discovered by base stations that sense terminals in their respective coverage areas.

A similar approach as in [19] has been taken by Amirijoo et al. [20], [21]. The authors though developed their solution for a different technology, namely, the Long Term Evolution (LTE) network, i.e., the cellular network that it is being designed to replace current UMTS networks. As in the case of [19], our work differs from [20], [21] in the way overlaps between cells are discovered. Furthermore, as a patent based on this work [22] claims, our work is intended to be more general and can be applied in the context of a range of technologies, including GSM, UMTS and LTE. The approaches described in [19] and [20], [21], as well as newer approaches such as [23], however, are based on technology-specific features and are, therefore, provide solutions only for those specific network technologies. 


\section{DISCUSSION}

a) Contribution: In this paper, we introduced a decentralized, three-layered framework for auto-configuring neighboring cell lists of radio access networks. As the main contribution of the paper, we presented DOC, a novel protocol that detects and continuously tracks the coverage overlaps among cells. By doing so, it maintains a distributed graph of overlapping cells. We evaluated the protocol through simulation, using realistic models for terminal mobility and radio coverage. We studied the system during its initialization phase, in steady state and during periods where addition or failure of base stations occur.

Note that DOC is a deterministic protocol and accurately computes the set of overlapping cells. While it uses Bloom filters to obtain an efficient probabilistic answer to which cells may overlap, it confirms or refutes such a probabilistic answer by computing the intersection of candidate sets in a deterministic manner. Due to the properties of Bloom filters, a probabilistic answer cannot contain false negatives.

Our evaluation shows that, given system characteristics such as size, terminal density and user mobility, one can dimension the protocol parameters, including the Bloom filter parameters, the distance between Bloom filters $D$, and threshold $H$, in order to achieve the design goals of low overhead and accuracy. The scalability experiments in Section IV-C show that the protocol overhead is likely to be acceptable for a deployment, within the parameters range studied. It further demonstrates the significance of proper dimensioning. For instance, the rapid increase in overhead for a growing terminal density is a consequence of using the same Bloom filter setting within the entire parameters range in which the terminal density increases tenfold. Regarding response times to external events, our experiments show that the protocol adapts within a few cycles to node addition, and it reacts to failures within a configurable time $t t l$. Also, the (additional) protocol overhead incurred by such events is minimal. The protocol can selfconfigure and reach steady state in very few cycles, even for systems with some 200 cells.

b) Controlling trade-offs: DOC has three key parameters for controlling the trade-off between overhead and various performance metrics. First, the control period $\tau$ controls the trade-off between protocol overhead and the timely detection of an overlapping cell relationship. As a complement, $t \mathrm{tl}$ controls the trade-off between the protocol overhead and the timely detection that such a relationship has disappeared. Finally, the threshold $H$ controls the trade-off between the protocol overhead and the level of connectivity of the graph of overlapping cells.

c) Using DOC for UMTS: Regarding the use of DOC in the context of UMTS networks, we note that the protocol uses information about the terminals that is typically available in devices of the access network. Therefore, the deployment effort of the protocol is limited to adding functionality to the management plane, without requiring changes to the control plane or the radio capabilities of network devices and user equipment. As referred to in Section V, the benefits of running DOC for radio access networks come at the cost of explicitly decoding the signals of all terminals in the range of each base station. In current UMTS implementations the only signals decoded by a base station are those coming from terminals that are being served by it. This is because the additional overhead for decoding all signals has no clear benefit to date. Moreover, involving a terminal in the uplink power control process of a cell that is not serving it to optimize its uplink signalto-interface ratio (SIR) might cause undesired interferences. However, DOC does not expect optimal uplink SIR from each terminal; during the initiation of a handover process, a terminal that is considered inside the destination cell is generally not seen with an optimal SIR from that cell. The overhead for decoding all signals could be an order of magnitude higher for DOC compared with current setups. Note though that our protocol requires decoding to compute a snapshot only at the beginning of each protocol cycle, and the overhead can therefore be controlled, e.g., by adjusting the length of this cycle.

d) Scalability: We conclude from our evaluation that DOC scales to systems of large size if the Bloom filters are dimensioned properly. In such a case, the protocol overhead from summary and ue-list messages is expected to remain constant when the system size grows, at the expense of an increasing size of the Bloom filters (i.e., the Summarymessage size) and an increase of the latency for discovering cell overlaps due to the increase in the height of the overlay spanning tree.

We believe it may be possible to adapt the protocol in such a way that its overhead becomes independent of both the system size and the message size, since the geographical distance between base stations limits the number of cells that potentially share a coverage area with any given cell in the network. One approach would be to use an overlay topology where neighboring nodes are geographically close to each other and to extend our protocol from one that executes on a tree to one that executes on a connected graph (the challenge here is to deal with cycles). An alternative approach would be, assuming each node knows its geographical position and taking into account the maximum range for each base station, to create an overlay that includes the graph of overlapping cells as a sub-graph. This can be achieved through an epidemic protocol, for instance T-MAN [24]. In such a case, the graph of overlapping cells can be created and maintained through each overlay node periodically exchanging the terminal list with its overlay neighbors. The overhead of such a simple protocol will be independent of the system size for most practical cases. Our protocol, DOC, does not assume that each node knows its geographical position, and, therefore, is applicable to settings where this information is difficult to acquire, for instance indoors (no GPS coverage) or in the case of mobile cells.

e) Automatic tuning of DOC parameters: The paper gives evidence that a proper dimensioning of the protocol parameters is essential to achieving low overhead. Dimensioning is traditionally performed during the planning phase, which, in the case of DOC, reduces the benefits of its self-configuration properties. Therefore, it is desirable to introduce mechanisms that automatically tune the protocol parameters during the operational phase. The Bloom filter size, for example, can be increased when the rate of false positives grows beyond an upper threshold and reduced when it falls below a lower 
threshold. For instance, [25] contains an analysis that shows how the probability of false positives can be kept constant when the ratio of filter size to filter members is maintained. To give a second example, parameter $\mathrm{H}$ in the paper is an absolute threshold that, if wrongly dimensioned, can cause under-detection of overlaps for those cells with a small number of active terminals. In this case, a self-configuration process can be considered by which $\mathrm{H}$ would be set automatically to a given percentage of the number of terminals in the cell. Furthermore, parameter D can be tuned automatically in order to keep the rate of summaries that are forwarded under a given threshold. Note that in each case mentioned one configuration parameter is being substituted by a different one and the total number of configuration parameters of the protocol stays the same. However, the new parameters are now independent of the network configuration and state.

f) Future work: In addition to the work outlined above, we consider further developing the protocol along the following lines. First, the protocol evaluation taught us that exchanging the ue-list messages between potential neighbors of the graph of overlapping cells is responsible for a significant part of the protocol overhead. The purpose of this exchange is to compute in a deterministic manner the graph of overlapping cells. An alternative would be to compute this graph in a probabilistic way, by using Bloom filters in the summary messages. We believe that such an approach would reduce the design complexity of the protocol, and, at the same time, reduce its overhead. In addition, we consider an alternative to detecting the disappearance of the overlapping relationship between cells. DOC currently uses a soft-state approach with a timeout of $t t l$. Our evaluation shows that the system can exhibit a high rate of changes in steady state, if $t t l$ is not properly dimensioned.

From the three layers of the architectural framework introduced in Section II, this paper focuses on the middle layer. We plan to develop functions for the other two layers as well: the construction of a suitable management overlay and, more importantly, the policy-based mechanism that configures the neighboring cell list for each cell. Finally, we plan to extend our framework from self-configuration of neighboring cells to include other autonomic functions, such as self-healing and self-optimization, which may involve dynamically configuring radio parameters.

\section{ACKNOWLEDGMENT}

The authors would like to thank Katarina Matusikova for her help with JavaSimulation and Harald Kallin for giving technical advice. Katarina is with the Ericsson Ireland Research Centre and Harald with Ericsson Research. Javier Baliosian has been partially funded by the European Community's Marie Curie Host Fellowships program at the Ericsson Ireland Research Centre.

\section{REFERENCES}

[1] J. Padgett, C. Gunther, and T. Hattori, "Overview of wireless personal communications," Commun. Mag., IEEE, vol. 33, no. 1, pp. 28-41, Jan. 1995.

[2] 3GPP , "TS 25.401, UTRAN overall description," 2007. [Online]. Available: http://www.3gpp.org/ftp/Specs/html-info/25401.htm
[3] J. Baliosian and R. Stadler, "Decentralized configuration of neighboring cells for radio access networks," World Wireless, Mobile Multimedia Netw., 2007. WoWMoM 2007. IEEE International Symp., pp. 1-6, June 2007.

[4] M. Dam and R. Stadler, "A generic protocol for network state aggregation," in RVK 05 RadioVetenskap och Kommunikation, Linkoping, Sweden, June 2005

[5] J. Baliosian, H. Oliver, A. Devitt, F. Sailhan, E. Salamanca, B. Danev, and G. Parr, "Self-configuration for radio access networks," in IEEE Policy 2006, 5-7 June 2006, p. 4.

[6] B. H. Bloom, Space/Time Trade-offs in Hash Coding with Allowable Errors, 1970, vol. 13, no. 7.

[7] P. Bose, H. Guo, E. Kranakis, and al., "On the falsepositive rate of bloom filters," 2004. [Online]. Available: http://cg.scs.carleton.ca/ jit/publications/

[8] K. Helsgaun, "Discrete event simulation in java," Roskilde University, Tech. Rep., 2000.

[9] J. Laiho, A. Wacker, and T. Novosad, Eds., Radio Network Planning and Optimisation for Umts. New York, NY, USA: John Wiley \& Sons, Inc., 2002.

[10] S. Miiller and H. Waller, "JMatLink," [Online]. Available: http://www.held-mueller.de/JMatLink/

[11] C. de Waal and M. Gerharz, "Bonnmotion. A mobility scenario generation and analysis tool." [Online]. Available: http://web.informatik.uni-bonn.de/IV/Mitarbeiter/dewaal/BonnMotion/

[12] A. Partow, "General purpose hash function algorithms." [Online]. Available: http://www.partow.net/programming/hashfunctions/

[13] ETSI, Universal Mobile Telecommunication System (UMTS), "Selection procedures for the choice of radio transmission technologies of the umts," UMTS 30.03 Version 3.2.0, 1998-04. [Online]. Available: http://www.3gpp.org/ftp/Specs/html-info/3003U.htm

[14] R. Chpudhury, S. Bandyopadhyay, and K. Paul, "A distributed mechanism for topology discovery in ad hoc wireless networks using mobile agents," in Mobile Ad Hoc Netw. Comput., 2000. MobiHOC. 2000 First Annual Workshop, 11 Aug. 2000, pp. 145-146.

[15] T. Clausen (Ed.), P. Jacquet (Ed.), C. Adjih, A. Laouiti, P. Minet, P. Muhlethaler, A. Qayyum, and L.Viennot, "Optimized link state routing protocol (olsr)," RFC 3626, Oct. 2003, Network Working Group. [Online]. Available: http://ietf.org/rfc/rfc3626.txt

[16] H. Olofsson, S. Magnusson, and M. Almgren, "A concept for dynamic neighbor cell list planning in a cellular system," in Proc. Seventh IEEE International Symp. Personal, Indoor Mobile Radio Commun. PIMRC'96, vol. 1, 15-18 Oct. 1996, pp. 138-142.

[17] S. Magnusson and H. Olofsson, "Dynamic neighbor cell list planning in a microcellular network," Universal Personal Commun. Record, 1997. Conf. Record., 1997 IEEE 6th International Conf., pp. 223-227 vol.1, Oct. 1997.

[18] F. Parodi, M. Kylvaja, G. Alford, J. Li, and J. Pradas, "An automatic procedure for neighbor cell list definition in cellular networks," in World Wireless, Mobile Multimedia Netw., 2007. WoWMoM 2007. IEEE International Symp., 18-21 June 2007, pp. 1-6.

[19] D. Soldani and I. Ore, "Self-optimizing neighbor cell list for utra fdd networks using detected set reporting," in Proc. VTC2007-Spring Veh. Technol. Conf. IEEE 65th, 22-25 Apr. 2007, pp. 694-698.

[20] M. Amirijoo, P. Frenger, F. Gunnarsson, H. Kallin, J. Moe, and K. Zetterberg, "Neighbor cell relation list and physical cell identity selforganization in LTE," in Commun. Workshops, 2008. ICC Workshops '08. IEEE International Conf., Beijing,, May 2008, pp. 37-41.

[21] —, "Neighbor cell relation list and measured cell identity management in LTE," in Netw. Operations Management Symp., 2008. NOMS 2008. IEEE, 7-11 Apr. 2008, pp. 152-159.

[22] J. Baliosian and R. Stadler, "Method of discovering overlapping cells," U.S. Patent WO/2008/151 674, Dec. 18, 2008.

[23] D. Huang, X. Wen, B. Wang, W. Zheng, and Y. Sun, "A self-optimising neighbor list with priority mechanism based on user behavior," in Comput., Commun., Control, Management, 2009. CCCM 2009. ISECS International Colloquium, vol. 3, Aug. 2009, pp. 144-147.

[24] M. Jelasity and O. Babaoglu, "T-man: Gossip-based overlay topology management," Eng. Self-Organising Syst., vol. 3910/2006, pp. 1-15, 2006. [Online]. Available: http://dx.doi.org/10.1007/11734697_1

[25] M. Mitzenmacher and E. Upfal, Probability and Computing: Randomized Algorithms and Probabilistic Analysis. New York, NY, USA: Cambridge University Press, 2005. 


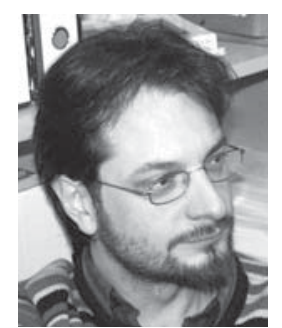

Javier Baliosian received the degree of Computer Engineer from the University of the Republic (Uruguay) in 1998, and his PhD from the Polytechnic University of Catalonia (Spain) in 2005. Dr. Baliosian have been working professionally since 1994 as a developer, designer, architect and leader of diverse distributed systems developments. In 2005 Dr. Baliosian obtained a Marie Curie Fellowship from the European Commission for a post-doctoral position at Ericsson Ireland where he worked as a researcher and project coordinator. Currently, he is an Assistant Professor at the University of the Republic in Uruguay.

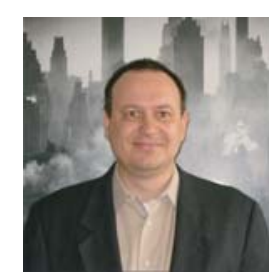

Rolf Stadler (www.ee.kth.se/ stadler) is professor at the Royal Institute of Technology (KTH) in Stockholm, Sweden. He holds an M.Sc. degree in mathematics and a $\mathrm{Ph} . \mathrm{D}$. in computer science from the University of Zurich. In 1991 he was a researcher at the IBM Zurich Research Laboratory. From 1992 to 1994 he was a visiting scholar at Columbia University in New York, which he joined in 1994 as a research scientist. From 1998 to 1999 he was a visiting professor at ETH Zurich. He joined the faculty of KTH in 2001 and is now at the School of Electrical Engineering, where he leads research in management of networked systems. 